\title{
Supplementary file for "Techno-Economic Feasibility of Reactive Distillation for Biodiesel Production from Algal Oil: Comparing with a Conventional Multiunit System"
}

Biswarup Mondal and Amiya K. Jana ${ }^{1}$

Energy and Process Engineering Laboratory, Department of Chemical Engineering, Indian Institute of Technology- Kharagpur, India-721302

Table S1. Group volume and area parameters for the UNIFAC model [54]

\begin{tabular}{cccc}
\hline Main group & Subgroup & $\begin{array}{c}\text { Volume parameter } \\
\left(R_{k}\right)\end{array}$ & $\begin{array}{c}\text { Area parameter } \\
\left(Q_{k}\right)\end{array}$ \\
\hline $\mathbf{C H}_{\mathbf{2}}$ & $\mathrm{CH}_{3}$ & 0.9011 & 0.848 \\
& $\mathrm{CH}_{2}$ & 0.6744 & 0.540 \\
& $\mathrm{CH}$ & 0.4469 & 0.228 \\
$\mathbf{O H}$ & $\mathrm{OH}$ & 1 & 1.2 \\
$\mathbf{C O O}$ & $\mathrm{COO}$ & 1.38 & 1.2 \\
\hline
\end{tabular}

Table S2. Group-group interaction parameters, $a_{m n}$, in Kelvin

\begin{tabular}{cccccc}
\hline & $\mathbf{C H}_{3}$ & $\mathbf{C H}_{2}$ & $\mathbf{C H}$ & $\mathbf{O H}$ & $\mathbf{C O O}$ \\
\hline $\mathbf{C H}_{3}$ & 0.00 & 0.00 & 0.00 & 986.5 & 387.1 \\
$\mathbf{C H}_{\mathbf{2}}$ & 0.00 & 0.00 & 0.00 & 986.5 & 387.1 \\
$\mathbf{C H}$ & 0.00 & 0.00 & 0.00 & 986.5 & 387.1 \\
$\mathbf{O H}$ & 156.4 & 156.4 & 156.4 & 0.0 & 190.3 \\
$\mathbf{C O O}$ & 529.0 & 529.0 & 529.0 & 88.63 & 0.0 \\
\hline
\end{tabular}

${ }^{1}$ Corresponding author. Tel.: +91 3222 283918; fax.: +91 3222 282250;

E-mail address: akjana@che.iitkgp.ac.in (A. K. Jana). 
Table S3. Cost estimating formula and parameter values

- Column shell

- Column tray

- Heat exchanger

- Reactor

$$
\text { Installed cost }(\$)=\left(\frac{M \& S}{280}\right) \times 937.636 \times D_{C}^{1.066} \times L_{C}^{0.802} \times\left(c_{i n}+c_{m}+c_{p}\right)
$$

where, $D_{C}=$ diameter of column $(\mathrm{m}), c_{i n} 2.18, c_{m}=3.67, c_{p}=1.0$ and $L_{C}$ $=$ height of the column expressed as: $L_{C}=0.4572 \times\left(N_{T}-1\right) \times 1.1$

$$
\text { Installed cost }(\$)=\left(\frac{M \& S}{280}\right) \times 97.243 \times D_{C}^{1.55} \times L_{T} \times\left(c_{s}+c_{t}+c_{m}\right)
$$

where, $c_{s}=1.0, c_{t}=0.0, c_{m}=1.7$ and $L_{T}=$ stack height of the tray, expressed as: $L_{T}=0.4572 \times\left(N_{T}-1\right)$

$$
\text { Installed cost }(\$)=\left(\frac{M \& S}{280}\right) \times 474.668 \times A^{0.65} \times\left(c_{i n}+\left(c_{d}+c_{p}\right) c_{m}\right)
$$

where, $c_{i n}=2.29, c_{m}=3.75, c_{p}=0.0$, and $c_{d}=0.8$ for condenser and 1.35 for reboiler. The heat transfer area of reboiler and condenser is given as:

$$
A=\frac{Q}{U_{H E} \Delta T_{L M T D}}
$$

Here, the overall heat transfer coefficient $\left(U_{H E}\right)$ is adopted as 1418.617 $\mathrm{W} /\left(\mathrm{m}^{2} . \mathrm{K}\right)$ for reboiler and $851.1699 \mathrm{~W} /\left(\mathrm{m}^{2} . \mathrm{K}\right)$ for condenser

Installed cost $(\$)=52920 \times D_{R}^{1.066} \times L_{R}^{0.802}$

where, $D_{R}=\left(3.977 \times 10^{-5} \times V_{R}\right)^{0.3333}$

and $L=2 D_{R} ; D_{R}=$ diameter, $L_{R}=$ height and $V_{R}=$ molar holdup of the reactor 